DOI https://doi.org/10.18551/rjoas.2017-02.02

\title{
M-ESTIMATORS FOR ASYMMETRIC PRICE TRANSMISSION MODELLING
}

\author{
Acquah De-Graft H., Associate Professor \\ Department of Agricultural Economics and Extension, University of Cape Coast, \\ Cape Coast, Ghana \\ E-mail: henrydegraftacquah@yahoo.com
}

\begin{abstract}
This paper introduces and compares M-estimators and OLS for modelling the Granger and Lee asymmetric price transmission model when the true data generating process is known. Results of 1000 Monte Carlo simulations, indicate that for normal data, the estimates of the coefficients of the Granger and Lee asymmetric price transmission model derived from the Least squares method as well as the $\mathrm{M}$ - and MM-estimation methods are accurate and equivalent to their true values. For data with outliers, estimates of the coefficients of the Granger and Lee asymmetric price transmission model derived using the Least squares method were inaccurate and different from their true values. In large samples, the Mestimation method produced accurate estimates that were equivalent to their true values in the presence of outliers. The MM-estimation method produced accurate estimates that were equivalent to their true values in the presence of outliers irrespective of sample size. These results indicate that the proposed $\mathrm{M}$ - and $\mathrm{MM}$-estimation techniques are likely to do no worse than the OLS with normal dataset and promise to do better when the dataset has outliers within the asymmetric price transmission modelling framework.
\end{abstract}

\section{KEY WORDS}

Monte Carlo Simulation, M-Estimation, MM-Estimation, Granger and Lee Asymmetry, Ordinary Least Squares Estimation, Outlier.

Granger and Lee (1989) proposed an econometric model to estimate asymmetric price transmission using the ordinary least squares (OLS) technique. Consequently, agricultural markets analysis has witnessed an explosion of the modelling of price transmission and asymmetries in agricultural commodity markets using the Granger and Lee asymmetric price transmission modelling approach and its variants.

However, the OLS technique used in estimating the Granger and Lee asymmetry has some limitations. For example, the OLS yields misleading result if its assumptions are not met. In practice, if assumptions of normality of the residuals are not met due to the presence of outliers, the OLS will produce misleading results in asymmetric price transmission analysis. Economic data (e.g. price series) used for asymmetric price analysis may contain outliers as commonly encountered in empirical research. However, the presence of outliers violate the assumption of the OLS estimation in the Granger and Lee asymmetric approach and its extensions.

In an empirical asymmetric price transmission analysis, Douglas (2010) notes that asymmetry detected is due to the presence of outliers in the data and that dropping the outliers in the data, he finds no evidence of asymmetry. The influence of outliers can be avoided by removing outliers from the database directly. However, Heckman (1979) notes that arbitrarily removal of some data from a database may lead to sample selection bias which can be considered as a specification error in linear regression. Apart from dropping the outliers in the data, influence of outliers can be reduced by transforming the data. Osborne and Overbay (2004) note that data transformation may be inappropriate for hypothesis testing and direct interpretation becomes difficult. Asymmetric price transmission researchers are concerned with obtaining the correct asymmetric adjustment parameters since they form the basis for detecting price asymmetry. But in the presence of outliers, the OLS may lead to bias estimates of the asymmetric adjustment parameters and an alternative will be to use robust regression approach such as $\mathrm{M}$ - and MM-estimation. Robust regression provides 
robust estimation even in the presence of outliers. Chatterjee and Hadi (2006) note that robust regression mimimizes the impact of outliers by giving smaller weight for outliers in the estimation procedure. The M-estimators remain robust to the presence of outliers as discussed in Huber (1973), Yohai (1987), Andrews et al. (1972), Bunke and Bunke (1986), Hampel et al. (1986), Lecoutre and Tassi (1987), Rousseeuw and Leroy (1987), Staudte and Sheather (1990), Rieder (1994), Jureckova and Sen (1996), Dodge and Jureckova (2000) and Jureckova and Picek (2006).

Empirically, very little research has been undertaken to introduce and compare MEstimators and OLS method for asymmetric price transmission models in the presence of outliers in the data. The purpose of this research is therefore to use Monte Carlo methods to investigate the performance of the OLS and M-estimators in estimating the Granger and Lee asymmetry using data with and without outliers.

The paper is organized as follows. The introduction is followed by the methods section which discusses the Granger and Lee Asymmetric model, Ordinary Least Squares (OLS) method and M-estimators. The results and discussion presents a practical application in which the performance of the OLS and M-Estimators in estimating true values of the Granger and Lee asymmetric data generating process is evaluated and the results of the Monte Carlo simulations are presented. Finally, the conclusion of the study is presented.

\section{METHODS OF RESEARCH}

Granger and Lee Asymmetry. Granger and Lee (1989) Error Correction Model data generating process can be specified as follows:

$$
\Delta y_{t}=\beta_{1} \Delta x_{t}+\beta_{2}(y-x)_{t-1}+\varepsilon_{1, t} \varepsilon_{1, t} \sim N\left(0, \delta^{2}\right)
$$

If $y$ and $x$ are price series integrated of the order one I (1) processes that are cointegrated, then there exists an equilibrium relationship between $y$ and $x$ which is defined by an error correction term. The long run equilibrium relationship captured by the error correction term are implicitly symmetric. Asymmetric adjustments can be introduced by splitting the error correction term as follows:

$$
\begin{aligned}
& (y-x)_{t}^{+}=\left[\begin{array}{c}
(y-x)_{t}, \text { if }(y-x)_{t}>0 \\
\text { zero otherwise }
\end{array}\right. \\
& (y-x)_{t}^{-}=\left[\begin{array}{c}
(y-x)_{t}, \text { if }(y-x)_{t}<0 \\
\text { zero otherwise }
\end{array}\right.
\end{aligned}
$$

The resulting Granger and Lee asymmetric model is specified as:

$$
\Delta y_{t}=\beta_{1} \Delta x_{t}+\beta_{2}^{+}(y-x)_{t-1}^{+}+\beta_{2}^{-}(y-x)_{t-1}^{-}+\varepsilon_{2, t} \varepsilon_{2, t} \sim N\left(0, \delta^{2}\right)
$$

In order to incorporate asymmetry, the speed of adjustment is allowed to differ for the positive and negative components of the Error Correction Term (ECT) since the long run relationship captured by the ECT are implicitly symmetric. Symmetry in eq. (4) is tested by determining whether the coefficients $\left(\beta_{2}{ }^{+}\right.$and $\left.\beta_{2}{ }^{-}\right)$are identical (that is $H_{0}: \beta_{2}{ }^{+}=\beta_{2}{ }^{-}$). The Granger and Lee Asymmetric Error correction model in eq. (4) can be represented as a standard regression model as specified below:

$$
y=X \beta+\varepsilon_{t} \varepsilon_{t} \square \operatorname{iidN}\left(0, \sigma^{2}\right)
$$

where: $\mathrm{y}$ is a response variable and independent variables in $\mathrm{X}$ are defined to include asymmetric adjustment terms. Subsequently, the estimation of parameters of the Granger 
and Lee Asymmetric model can be done using Ordinary Least Squares method and Mestimators and the results compared.

Ordinary Least Squares Estimation (OLS). In the ordinary least squares estimation, a matrix $X$, vectors $Y$ and $\epsilon$ are defined as:

$$
X=\left(\begin{array}{ccc}
x_{11} & \cdots & x_{1 p} \\
\vdots & \ddots & \vdots \\
x_{n 1} & \cdots & x_{n p}
\end{array}\right)=\left(\begin{array}{c}
x_{1}^{T} \\
\vdots \\
x_{n}^{T}
\end{array}\right), Y=\left(\begin{array}{c}
y_{1} \\
\vdots \\
y_{n}
\end{array}\right) \text { and } \in=\left(\begin{array}{c}
\epsilon_{1} \\
\vdots \\
\epsilon_{n}
\end{array}\right)
$$

Given the classic model $Y=X \beta+\epsilon$, then it can be estimated that the least square aims to minimize:

$$
\begin{gathered}
\sum_{i=1}^{n} \in_{1}^{2}=\in^{T} \in \\
(Y-X \beta)^{T}(Y-X \beta) \\
Y^{T} Y-Y^{T} X \beta-\beta^{T} X^{T} Y+\beta^{T} X^{T} X \beta
\end{gathered}
$$

At minimum,

$$
\begin{aligned}
\frac{\partial}{\partial \beta}\left(\sum_{i=1}^{n} \epsilon_{1}^{2}\right) & =\frac{\partial}{\partial \beta}\left(Y^{T} Y-Y^{T} X \beta-\beta^{T} X^{T} Y+\beta^{T} X^{T} X \beta\right) \\
& =0-X^{T} Y-X^{T} Y+2\left(X^{T} X\right) \beta
\end{aligned}
$$

Hence, the least square estimator $\hat{\beta}$ is the solution:

$$
X^{T} X \widehat{\beta}=X^{T} Y
$$

When $X^{T} X$ is non-singular and the minimisation is given as $\hat{\epsilon}^{T} \hat{\epsilon}=\sum_{i=1}^{n} r_{1}^{2}$, then the least square estimator can be evaluated directly from the data as:

$$
\hat{\beta}=\left(X^{T} X\right)^{-1} X^{T} Y
$$

M-Estimator. Huber (1973) developed a group of maximum likelihood estimators referred to as the M-estimator. The M-estimation technique is one of the most common method of robust regression and is efficient as the OLS. M-estimator aims at minimizing a function $\rho$ of the errors. The objective function of the M-estimate is stated as:

$$
\sum_{i=1}^{n} \rho\left(\frac{e_{i}}{s}\right)=\sum_{i=1}^{n} \rho\left(\frac{y_{i}-x^{T} \beta}{s}\right)
$$

"s" in eq. (9) is an estimate of scale from a linear combination of the residuals. The function $\rho$ in eq. (9) provides the contribution of each residual to the objective function. Alma (2011) posits that a reasonable $\rho$ should have in it the following properties:

$$
\begin{gathered}
\rho(e) \geq 0 \\
\rho(0)=0 \\
\rho(e)=\rho(-e) \\
\boldsymbol{\rho}\left(\boldsymbol{e}_{\boldsymbol{i}}\right) \geq \boldsymbol{\rho}\left(\dot{\boldsymbol{e}}_{\imath}\right) \text { for }\left|e_{i}\right| \geq\left|\dot{e}_{l}\right|, \text { and } \rho \text { is continuous }
\end{gathered}
$$

Given least square estimation, $\rho\left(e_{t}\right)=e_{i}^{2}$, the system of normal equations can be solve from the above function by taking partial derivatives with respect to $\beta$ while setting the function to 0 . Minimizing eq. (9) with respect to each of the parameters, $\beta_{1}, \ldots, \beta_{p}$, yields the following system of $p$ equations: 


$$
\sum_{i=1}^{n} x_{i j} \varphi\left(\frac{e_{i}}{s}\right)=\sum_{i=1}^{n} x_{i j} \varphi\left(\frac{y_{i}-x_{i}^{T} \beta}{s}\right)=0 ; j=1,2, \ldots, p \text { and } i=1,2, \ldots, p
$$

where: $\varphi(u)=\frac{\partial \rho}{\partial \rho}$ is the score function. A 'weight' function is then defined as:

$$
\omega(u)=\frac{\varphi(u)}{u}
$$

This yields $\omega_{i}=\omega\left(\frac{\epsilon_{i}}{s}\right)$ for $\mathrm{i}=1, \ldots, \mathrm{n}$, with $\omega_{i}=1$ if $\epsilon_{i}=0$. Substituting it into eq. (10) yields:

$$
\begin{gathered}
\sum_{i=1}^{n} x_{i j} w_{i} \frac{\epsilon_{i}}{s}=\sum_{i=1}^{n} x_{i j} w_{i}\left(y_{i}-x_{i}^{T} \beta\right) \frac{1}{s}=0 j=1, \ldots, p \\
\rightrightarrows \sum_{i=1}^{n} x_{i j} w_{i}\left(y_{i}-x_{i}^{T} \beta\right)=0 j=1, \ldots, p \\
\rightrightarrows \sum_{i=1}^{n} x_{i j} w_{i} x_{i} \beta=\sum_{i=1}^{n} x_{i j} w_{i} y_{i} j=1, \ldots, p
\end{gathered}
$$

Since $s \neq 0$, the weight matrix $W=\operatorname{diag}\left(\left\{w_{i}: i=1, \ldots, n\right\}\right)$ can be defined as follows:

$$
W=\left(\begin{array}{ccc}
w_{i} & \ldots & 0 \\
\vdots & w_{2} & \vdots \\
0 & \ldots & w_{n}
\end{array}\right)
$$

The results in the matrix from of equation (11) yields:

$$
\begin{gathered}
X^{T} W X \beta=X^{T} W Y \\
\hat{\beta}=\left(X^{T} W X\right)^{-1} X^{T} W Y
\end{gathered}
$$

Eq. (12) is very similar to the solution for the least square estimator only that it differs from the least square estimator with the introduction of a weight matrix. The weight matrix in eq. (12) reduces the influence of outliers.

For all M-estimators (i.e. M-and MM-estimators), when the errors are normally distributed, their asymptotic variance is given by:

$$
V(\varphi, \Phi)=\hat{\sigma}^{2} \frac{A(\varphi, \Phi)}{B^{2}(\varphi, \Phi)}
$$

The variance-covariance matrix of the estimated regression coefficient as:

$$
V(\hat{\beta})=\hat{\sigma}^{2} \frac{A(\varphi, \Phi)}{B^{2}(\varphi, \Phi)}\left(X^{T} X\right)^{-1}
$$

Through the iteration process, $A(\varphi, \Phi)=E\left(\varphi^{2}, \Phi\right)$ and $B^{2}(\varphi, \Phi)=\left(E\left(\varphi^{\prime}, \Phi\right)\right)^{2}$.

$M M$ - estimator. The MM-estimator, according to Yohai (1987) is a special type of Mestimation which is a combination of high breakdown of estimation values and efficient estimation. Yohai (1987) describes three stages that defines MM-estimator:

1. A high breakdown estimator is used to find an initial estimate, which we denote $\bar{\beta}$. The estimator need not be efficient. Using this estimate, the residual, $r_{i}(\bar{\beta})=y_{i}-$ $x_{i}^{T} \bar{\beta}$ are computed. 
2. Using these residuals from robust fit, an M-estimate of scale with $50 \%$ Breakdown point (BDP) is computed. This $s\left(r_{1}(\bar{\beta}), \ldots, r_{n}(\bar{\beta})\right)$ is denoted $s_{n}$. The objective function used in this state is labelled $\rho_{0}$.

3. The MM-estimator is now defined as an M-estimator of $\beta$ using a redescending score function, $\varphi_{1}(u)=\frac{\partial \rho 1(u)}{\partial u}$, and the scale estimate $s_{n}$ obtained from stage 2. So an MMestimator $\hat{\beta}$ is defined as a solution to

$$
\sum_{i=1}^{n} x_{i j} \varphi_{1}\left(\frac{y_{i}-x_{i}^{T} \beta}{s_{n}}\right)=0 j=1, \ldots, p .
$$

The objective function $\rho_{1}$ associated with this score function do not have to be the same as $\rho_{0}$ but it must satisfy the following conditions:

a. $\rho$ is symmetric and continuously differentiable, and $\rho(0)=0$;

b. There exists $a>0$ such that $\rho$ is strictly increasing on $[0, a]$ and constant on $[a, \infty]$;

c. $\rho_{1}(u) \leq \rho_{0}(u)$.

A final condition that must be satisfied by the solution to equation (15) is that:

$$
\sum_{i=1}^{n} x_{i j} \varphi_{1}\left(\frac{y_{i}-x_{i}^{T} \widehat{\beta}}{s_{n}}\right) \leq \sum_{i=1}^{n} x_{i j} \varphi_{1}\left(\frac{y_{i}-x_{i}^{T} \widetilde{\beta}}{s_{n}}\right)
$$

It is clear from the equations that the third stage is just M-estimation with an extra condition on the solution.

\section{RESULTS AND DISCUSSION}

Comparison of M-Estimators and OLS for Granger and Lee Asymmetry. In order to compare the performance of the M-estimators with the OLS in estimation of an asymmetric price transmission model a series of Monte Carlo Simulations are conducted. The simulation is based on the asymmetric data generation process specified in eq. (4) and the data is simulated from the Granger and Lee asymmetric error correction model as follows:

$$
\Delta y_{t}=0.7+0.5 \Delta x_{t}-0.25\left(y_{t}-x_{t}\right)_{t-1}^{+}-0.75\left(y_{t}-x_{t}\right)_{t-1}^{-}+\varepsilon
$$

$y_{t}$ and $x_{t}$ are generated as I (1) non-stationary variables that are cointegrated. The error correction terms $\left(\left(y_{t}-x_{t}\right)^{+}{ }_{t-1},\left(y_{t}-x_{t}\right)^{-}{ }_{t-1}\right)$ denote the positive and negative deviations from the equilibrium relationship between $y_{t}$ and $x_{t}$. For the normal data, the errors are generated from a normal distribution with a mean 0 and a variance of $1(\varepsilon \square N(0,1))$. In order to create outliers in the data, nine observations of the errors generated for the normal data with values generated from a normal distribution with a mean of 0 and a variance of 1 , were replaced with nine observations from the normal distribution with a mean of 20 and variance of $1(\varepsilon \square N(20,1))$.

Using 1000 Monte Carlo simulations, the performance of M estimators and the OLS in estimating the parameters of Granger and Lee asymmetric price transmission model (data generating process) is investigated under conditions of different sample sizes and asymmetry given by $\left(\beta_{2}^{+}, \beta_{2}^{-}\right) \in\left(\beta_{2}, \beta_{3}\right) \in(-0.25,-0.75)$ for the normal data as well as the data with outliers. This study draws from Cook et al $(1999,2000)$ and Acquah $(2012$, 2013) in assigning the asymmetric adjustment parameters $\left(\beta_{2}{ }^{+}, \beta_{2}{ }^{-}\right)$.

When an estimator performs well, then the averaged value of estimates of each parameter should be close to their true values in the data generating process. As shown in Table 1, in the absence of outliers, all the three methods performed well, with the averaged 
estimates all nearly equal or close to their true values of $\beta_{0}=0.7, \beta_{1}=0.5, \beta_{2}=-0.25, \beta_{3}=-0.75$ in the various sample size.

The results are consistent with Muthukrishnan and Radha (2010) who noted that Mestimators yields the same results as the least squares for normal data in a simple linear regression analysis. Similarly, Ryan (1997) asserts that robust methods such as M and MM estimation methods performs almost as well as the OLS when the data is free of mistakes and influential data points.

Table 1 - Normal Data (Without Outliers)

\begin{tabular}{|c|c|c|c|c|c|c|}
\hline \multirow[t]{3}{*}{ Sample Size } & \multirow[t]{3}{*}{ Properties of Data } & \multirow[b]{2}{*}{ Method } & \multicolumn{4}{|c|}{ Estimates } \\
\hline & & & $\beta_{0}$ & $\beta_{1}$ & $\beta_{2}$ & $\beta_{3}$ \\
\hline & & OLS & 0.70 & 0.50 & -0.25 & -0.75 \\
\hline \multirow[t]{2}{*}{$N=50$} & \multirow[t]{2}{*}{ Normal } & M-Estimation & 0.70 & 0.50 & -0.25 & -0.75 \\
\hline & & MM-Estimation & 0.71 & 0.50 & -0.25 & -0.74 \\
\hline \multirow[t]{3}{*}{ Sample Size } & \multirow[t]{3}{*}{ Properties of Data } & \multirow[b]{2}{*}{ Method } & \multicolumn{4}{|c|}{ Estimates } \\
\hline & & & $\beta_{0}$ & $\beta_{1}$ & $\beta_{2}$ & $\beta_{3}$ \\
\hline & & OLS & 0.70 & 0.50 & -0.25 & -0.75 \\
\hline \multirow[t]{2}{*}{$N=150$} & \multirow[t]{2}{*}{ Normal } & M-Estimation & 0.70 & 0.50 & -0.25 & -0.74 \\
\hline & & MM-Estimation & 0.70 & 0.50 & -0.25 & -0.74 \\
\hline \multirow[t]{3}{*}{ Sample Size } & \multirow[t]{3}{*}{ Properties of Data } & & \multicolumn{4}{|c|}{ Estimates } \\
\hline & & Method & $\beta_{0}$ & $\beta_{1}$ & $\beta_{2}$ & $\beta_{3}$ \\
\hline & & OLS & 0.70 & 0.50 & -0.25 & -0.75 \\
\hline \multirow[t]{2}{*}{$N=500$} & \multirow[t]{2}{*}{ Normal } & M-Estimation & 0.70 & 0.50 & -0.25 & -0.75 \\
\hline & & MM-Estimation & 0.71 & 0.50 & -0.25 & -0.75 \\
\hline
\end{tabular}

Note: based on 1000 Monte Carlo Simulation.

In the presence of outliers, very different results are produced. In small and moderate samples of 50 and 150 respectively, the MM-estimator outperforms the M-estimator which in turn outperforms the OLS estimator. As shown in Table 2, OLS estimator performs poorly with its parameter estimates entirely different from the true parameter values of $\beta_{0}=0.7, \beta_{1}=0.5, \beta_{2}=-0.25, \beta_{3}=-0.75$ as defined in the data generating process. On the other hand, the MM-estimators perform very well in small and moderate samples with its parameter estimates close to their true values. In comparison to the OLS, the M-estimator performs reasonably well in small and moderate samples with a few estimates slightly different from their true values.

Table 2 - Data with Outliers

\begin{tabular}{|c|c|c|c|c|c|c|}
\hline \multirow[t]{3}{*}{ Sample Size } & \multirow[t]{3}{*}{ Properties of Data } & \multirow[b]{2}{*}{ Method } & \multicolumn{4}{|c|}{ Estimates } \\
\hline & & & $\beta_{0}$ & $\beta_{1}$ & $\beta_{2}$ & $\beta_{3}$ \\
\hline & & OLS & 3.00 & 0.52 & -0.16 & -1.14 \\
\hline \multirow[t]{2}{*}{$N=50$} & With Outliers & M-Estimation & 0.93 & 0.50 & -0.25 & -0.78 \\
\hline & & MM-Estimation & 0.73 & 0.50 & -0.27 & -0.73 \\
\hline \multirow[t]{3}{*}{ Sample Size } & Properties of Data & & \multicolumn{4}{|c|}{ Estimates } \\
\hline & & Method & $\beta_{0}$ & $\beta_{1}$ & $\beta_{2}$ & $\beta_{3}$ \\
\hline & & OLS & 1.00 & 0.49 & -0.44 & -2.00 \\
\hline \multirow[t]{2}{*}{$N=150$} & With Outliers & M-Estimation & 0.73 & 0.50 & -0.27 & -0.85 \\
\hline & & MM-Estimation & 0.70 & 0.50 & -0.25 & -0.75 \\
\hline \multirow[t]{3}{*}{ Sample Size } & Properties of Data & & \multicolumn{4}{|c|}{ Estimates } \\
\hline & & Method & $\beta_{0}$ & $\beta_{1}$ & $\beta_{2}$ & $\beta_{3}$ \\
\hline & & OLS & 1.01 & 0.50 & -0.26 & -0.52 \\
\hline \multirow[t]{2}{*}{$N=500$} & With Outliers & M-Estimation & 0.72 & 0.50 & -0.25 & -0.73 \\
\hline & & MM-Estimation & 0.70 & 0.50 & -0.25 & -0.75 \\
\hline
\end{tabular}

Note: based on 1000 Monte Carlo Simulation.

In large samples of 500 contaminated with outliers, the M-and MM-estimators perform well with their parameter estimates identical to their true values whilst the OLS performs poorly with estimates different from their true values. 
From Table 1, it is clear that the results of the M-and MM-estimators are similar to the least squares and close to their true values in the data without outliers. However, in the data with outliers (Table 2), the least squares method is affected by outliers in small, moderate and large samples. The M-estimator remains robust to outliers in large samples whilst the MM-estimator yielded reasonable estimates of the asymmetric price transmission data generating process in the various sample sizes ( small, moderate, large) of data with and without outliers. The results are consistent with Ryan (1997) assertion that robust methods such as $\mathrm{M}$ and MM estimation methods perform much better than OLS when the data has outliers. Similarly, Muthukrishnan and Radha (2010) noted that when outliers are present in the data, OLS performs poorly and does not provide useful information in a simple linear regression analysis. However, M-estimators are robust to outliers and provides useful and accurate information in a simple linear regression analysis.

\section{CONCLUSION}

The performance of M-estimators have been investigated in asymmetric price transmission regression modelling. The findings indicate that the $\mathrm{M}$ - and $\mathrm{MM}$-estimators yield the same result as the OLS with normal data. However, when outliers are present in the data, the least squares do not provide useful information in small, moderate and large samples of data. On the other hand, the MM-estimators provide useful information and are not affected by outliers as sample size increases from small to large. The M-estimator remains robust to outliers in large samples. In summary, the results of the simulation indicate that the M-estimators can be considered in asymmetric price transmission modelling and may do better than the OLS when the data contains outliers.

\section{REFERENCES}

1. Acquah, H. D. (2012). A bootstrap approach to testing for symmetry in the Granger and Lee Asymmetric Error Correction Model. Russian Journal of Agricultural and SocioEconomic Sciences, 11(11): 33-36.

2. Acquah, H. D. (2013): Using bootstrap method to evaluate the power of tests for nonlinearity in asymmetric price relationship. Journal of Economics and Behavioral Studies, 5, (4), 237-241.

3. Alma, O. G. (2011). Comparison of robust regression method in linear regression. Int. J. Contemp. Math. Sciences, 6(9), $409-421$.

4. Andrews, D. F., Bickel, P. J., Hampel, F. R., Huber, P. J., Rogers, W. H., \& Tukey, J.W. (1972). Robust estimates of location - Survey and advances. New Jersey: Princeton University Press.

5. Bunke, H., \& Bunke, O. (Eds.). (1986). Statistical inference in linear models. Chichester, U.K: John Wiley \& Sons.

6. Chatterjee, S., \& Hadi, A. S. (2006). Regression analysis by example. New Jersey: John Wiley \& Sons.

7. Cook, S., Holly, S., \& Turner, P. (1999). The Power of tests for non-linearity: the case of Granger-Lee asymmetry, Economics Letters, 62, pp.155-159.

8. Cook, S., Holly, S., \& Turner, P. (2000). The Power of Tests for Non-linearity: The Escribano-Pfann Model, Computational Economics, 15, pp. 223-226.

9. Dodge, Y., \& Jureckova, J. (2000). Adaptive regression. New York: Springer Science \& Business Media.

10. Douglas, C. C. (2010). Do gasoline prices exhibit asymmetry? Not usually!. Energy Economics, 32(4), 918-925.

11. Granger, C. W. J., \& Lee, T. H. (1989). Investigation of production, sales and inventory relationships using multicointegration and non $\square$ symmetric error correction models. Journal of Applied Econometrics, 4(1), 145-159.

12. Hampel, F. R., Ronchetti, E. M., Rousseeuw, P. J., \& Stahel, W. A. (1986). Robust statistics: The approach based on influence functions. New York: John Wiley and Sons. 
13. Heckman, J. J. (1979). Sample selection bias as a specification error. Econometrica, 47, 153-161.

14. Huber, P. J. (1973). Robust regression: Asymptotics, conjectures and Monte Carlo. The Annals of Statistics, 1(5), 799-821.

15. Jureckova, J., \& Picek, J. (2006). Robust statistical methods with R. Florida: Chapman \& Hall/CRC.

16. Lecoutre, J. P., \& Tassi, P. (1987). Statistique non parametricque et robustesse. Paris: Economica Pub.

17. Muthukrishnan, M., \& Radha, M (2010). M-estimators in regression models. Journal of Mathematics Research, 2 (4), 23

18. Osborne, J. W., \& Overbay, A. (2004). The power of outliers (and why researchers should always check for them). Practical Assessment, Research \& Evaluation, 9(6), 1-8.

19. Rieder, H. (1994). Robust asymptotic statistics. New York: Springer.

20. Ryan, T. P. (1997). Modern regression methods. New York, NY: John Wiley \& Sons, Inc.

21. Rousseeuw, P. K., \& Leroy, A. M. (1987). Robust regression and outlier detection. New York: John Wiley \& Sons.

22. Staudte, W. A., \& Sheather, S. J. (1990). Robust estimation and testing. New York, NY: John Wiley \& Sons.

23. Yohai, V. J. (1987). High breakdown-point and high efficiency robust estimates for regression. The Annals of Statistics, 15, 642-656. 